\title{
CORRECTION TO THE PAPER ON GLOBAL CONVERGENCE \\ OF A CLASS OF TRUST REGION ALGORITHMS FOR OPTIMIZATION WITH SIMPLE BOUNDS*
}

\author{
A. R. CONN†, N. I. M. GOULD , AND PH. L. TOINT§
}

\begin{abstract}
A correction is made to the paper entitled "Global convergence of a class of trust region algorithms for optimization with simple bounds" [Conn, Gould, and Toint, SIAM J. Numer. Anal., 25 (1988), pp. 433-460]. First, an error is pointed out in the proof of the fact that the correct set of active bounds is determined after a finite number of iterations. A new proof of the relevant theorem is given.
\end{abstract}

Key words. trust regions, convergence theory, optimization with bounds

AMS(MOS) subject classifications. primary $65 \mathrm{~K} 05$; secondary $90 \mathrm{C} 30$

The purpose of the present note is not to reconsider the complete problem of global convergence and active set determination for the class of trust region algorithms considered in [1], but rather to correct an error in this paper. Good knowledge of the contents of [1] will therefore be assumed, and reference to this paper will be frequent. In particular, all notation and definitions will be borrowed from [1]. We will also adopt the convention that equation numbers between braces refer to equations of [1].

1. The error. The error we want to correct occurs in the proof of Theorem 14. Towards the end of the proof equation $\{169\}$ is used to deduce $\{176\}$. This deduction is incorrect because $\{169\}$ only holds for successful iterates, that is for $k \in S$, and thus cannot be applied for all $k$. As a consequence, $\{176\}$, and hence $\{177\}$, only holds for successful iterates, which is not enough to obtain the desired contradiction.

2. A new proof of Theorem 14.

TheOrem 14. Assume (AS.1)-(AS.7) and $\{155\}$ hold. Then,

$$
I\left(x_{k}\right)=I\left(x_{*}\right)
$$

for $k$ sufficiently large, where $x_{*}$ is arbitrary in $L$.

Proof. First choose an arbitrary limit point $x_{*} \in L$, say, and denote by $X_{*}$ the connected set of limit points to which $x_{*}$ belongs, according to Lemma 12. Note that Theorem 11 implies that $x_{*}$ is critical. Since $X_{*}$ is connected, it is possible to find a $\psi>0$ such that the distance from $X_{*}$ to any other limit point not in $X_{*}$ is bounded below by $\psi$. As in Lemma 13 , we can choose $\delta \in\left(0, \frac{1}{4} \psi\right]$ sufficiently small and a $k_{1}$ sufficiently large to guarantee that, for all $x \in \mathcal{N}\left(X_{*}, 2 \delta\right)$,

$$
\begin{gathered}
I(x) \subseteq I\left(X_{*}\right), \\
\operatorname{sgn}\left([\nabla f(x)]_{j}\right)=\operatorname{sgn}\left(\left[\nabla f\left(x_{*}\right)\right]_{j}\right) \quad \text { and }\left|[\nabla f(x)]_{j}\right| \geqq \varepsilon_{1}
\end{gathered}
$$

with

$$
\varepsilon_{1} \stackrel{\text { def }}{=} \min _{x_{*} \in X_{*}} \min _{j \in I\left(X_{*}\right)}\left|\left[\nabla f\left(x_{*}\right)\right]_{j}\right|>0
$$

\footnotetext{
* Received by the editors June 8, 1988; accepted for publication September 18, 1988.

$\uparrow$ Department of Combinatorics and Optimization, University of Waterloo, Waterloo, Canada.

\$ Harwell Laboratories, Harwell, United Kingdom.

$\S$ Department of Mathematics, Facultés Universitaires Notre-Dame de la Paix, B-5000 Namur, Belgium.
} 
the sequence $\left\{I\left(x_{k}\right)\right\}$ is nondecreasing (according to Lemma 13) and that

$$
d\left(x_{k}, X_{*}\right)>\delta \Rightarrow d\left(x_{k}, X_{*}\right) \geqq \frac{1}{2} \psi
$$

for all $k \geqq k_{1}$. We now define the subsequence $\left\{k_{j}\right\}$ as

$$
\left\{k_{j}\right\}=\left\{k \geqq k_{1} \mid x_{k} \in \mathcal{N}\left(X_{*}, \delta\right) \text { and } k \in S\right\},
$$

where, as in [1], $S$ denotes the set of indices of successful iterations. By definition of $X_{*}$, the subsequence (6) has infinitely many terms. For future reference, we note here that $(2),(6)$, and the nondecreasing character of $\left\{I\left(x_{k}\right)\right\}$ imply that

$$
I\left(x_{k_{j}}+s_{k_{j}}\right) \subseteq I\left(X_{*}\right)
$$

for all $j$.

Assume now, for the purpose of obtaining a contradiction, that, for all $j$,

$$
I\left(x_{k_{j}}+s_{k_{j}}\right) \subset I\left(X_{*}\right),
$$

where the inclusion is strict. Because of $\{155\}$, there must be a nonempty set $T \subseteq I\left(X_{*}\right)$ such that $T \cap I\left(x_{k_{j}}^{C}\right)$ is empty for all $j$. Therefore, from (AS.5), (AS.7), and (3), we may deduce that, for all $j$,

$$
\left[a_{k_{j}}^{s}\right]^{2} \geqq \frac{1}{\sigma_{2}^{2}} \sum_{t \in T}\left[g_{k_{j}}\right]_{t}^{2} \geqq \frac{\varepsilon_{1}^{2}}{\sigma_{2}^{2}} \stackrel{\text { def }}{=} \varepsilon^{2} .
$$

Lemma 6 then implies that

$$
b_{k_{j}}\left[f\left(x_{k_{j}}\right)-f\left(x_{k_{i}+1}\right)\right] \geqq \frac{1}{2} \mu c_{3} \varepsilon^{2} \min \left[\varepsilon^{2}, b_{k_{j}} \Delta_{k_{j}}\right],
$$

and (AS.6) thus gives that

$$
\lim _{j \rightarrow \infty} b_{k_{j}} \Delta_{k_{j}}=0 .
$$

The inequality $b_{k_{j}} \geqq 1$, (AS.3), and (11) show that

$$
\left\|s_{k_{j}}\right\| \leqq \sigma_{1} \beta_{2} \Delta_{k_{j}} \leqq \frac{1}{2} \delta
$$

for $j$ larger than $j_{1} \geqq 1$, say. Then, for all $j \geqq j_{1}$,

$$
d\left(x_{k_{j}+1}, X_{*}\right) \leqq d\left(x_{k_{i}}, X_{*}\right)+\left\|s_{k_{j}}\right\| \leqq \frac{3 \delta}{2} \leqq \frac{3}{8} \psi,
$$

because of the definition (6). Hence, because of (5), the iterates cannot jump outside $\mathcal{N}\left(X_{*}, \delta\right)$, and we must have that $x_{k_{+}+1} \in \mathcal{N}\left(X_{*}, \delta\right)$ again. This implies that the next successful iterate belongs to $\mathcal{N}\left(X_{*}, \delta\right)$. Therefore, the subsequence $\left\{k_{j}\right\}$ is identical to the complete sequence of successful iterates with $k \geqq k_{j_{1}}$. Hence we may deduce from (11) that

$$
\lim _{\substack{k \rightarrow \infty \\ k \in S}} b_{k} \Delta_{k}=0 .
$$

But the mechanism of the algorithm then implies that

$$
\lim _{k \rightarrow \infty} \Delta_{k}=0 .
$$

As a consequence of this limit and of $\{10\}$ and $\{13\}$, we may increase the value of $k_{1}$, if necessary, to ensure that $x_{k}, x_{x}^{C}$ and $x_{k}+s_{k}$ all belong to $\mathcal{N}\left(X_{*}, 2 \delta\right)$ for all $k \geqq k_{1}$, and therefore that (2) and (3) hold at these points. 
We now wish to show that all iterations are successful for $k$ sufficiently large. Assume this is not the case. It is then possible to find an index $k$ arbitrarily large such that

$$
k \notin S \text { and } k+1 \in S \text {. }
$$

Note that, because of $\{24\}$ and $\{30\}$, we have that

$$
b_{k} \Delta_{k} \leqq \frac{1}{\gamma_{0}} b_{k+1} \Delta_{k+1}
$$

and therefore that (14) implies that $b_{k} \Delta_{k}$ is arbitrarily small for $k$ large enough and satisfying (16). In particular, for such a $k$,

$$
b_{k} \Delta_{k}<\varepsilon^{2} \min \left[1, \frac{c_{3} \gamma_{0}^{2}(1-\mu)}{2 \beta_{2}^{2}\left(c_{5} \sigma_{1}^{2}+1\right)}\right] \text {. }
$$

Now, if we have that

$$
I\left(x_{k}^{C}\right) \subset I\left(X_{*}\right),
$$

with strict inclusion, then we may deduce from (AS.5), (AS.7), and (3) that,

$$
\left[a_{k}^{s}\right]^{2} \geqq \varepsilon^{2},
$$

as for (9). Using this equation, (18), and Lemma 6, we obtain that for $k$ sufficiently large,

$$
f\left(x_{k}\right)-m_{k}\left(x_{k}+s_{k}\right) \geqq \frac{1}{2} c_{3} \varepsilon^{2} \Delta_{k} .
$$

Since $b_{k} \geqq 1$, the inequalities $\{107\}$ and (21) now imply that

$$
\left|\rho_{k}-1\right| \leqq \frac{\beta_{2}^{2}\left(c_{5} \sigma_{1}^{2}+1\right)}{c_{3} \varepsilon^{2}} b_{k} \Delta_{k}
$$

for $k$ sufficiently large. The bound (18) and $\gamma_{0}<1$ then yield that $\rho_{k}>\mu$ for $k$ large enough. But this implies that $k \in S$, which is impossible because of the first part of (16). Hence (19) cannot hold, and (2) taken at $x_{k}^{C}$ yield that $I\left(x_{k}^{C}\right)=I\left(X_{*}\right)$ for $k$ large enough and satisfying (16). Condition $\{155\}$ and (2) taken at $x_{k}+s_{k}$ then imply that, for such a $k$,

$$
I\left(x_{k}+s_{k}\right)=I\left(X_{*}\right) .
$$

Now observe that, since $k \notin S,\{6\}$ and $\{23\}$ imply that

$$
\begin{aligned}
m_{k+1}\left(x_{k+1}+s_{k+1}\right)-m_{k}\left(x_{k}+s_{k}\right) & =m_{k+1}\left(x_{k}+s_{k+1}\right)-m_{k}\left(x_{k}+s_{k}\right) \\
& =g_{k}^{T}\left(s_{k+1}-s_{k}\right)+\frac{1}{2}\left[s_{k+1}^{T} B_{k+1} s_{k+1}-s_{k}^{T} B_{k} s_{k}\right] .
\end{aligned}
$$

Now decomposing $g_{k}$ and $\left(s_{k+1}-s_{k}\right)$ as

$$
g_{k}=g_{k}^{R}+g_{k}^{N} \text { and } s_{k+1}-s_{k}=\left(s_{k+1}-s_{k}\right)^{R}+\left(s_{k+1}-s_{k}\right)^{N} \text {, }
$$

where $g_{k}^{N}$ and $\left(s_{k+1}-s_{k}\right)^{N}$ belong to $C\left(x_{*}\right)$ and where $g_{k}^{R}$ and $\left(s_{k+1}-s_{k}\right)^{R}$ belong to $C\left(x_{*}\right)^{\perp}$, we write that

$$
g_{k}^{T}\left(s_{k+1}-s_{k}\right)=\left[g_{k}^{R}\right]^{T}\left(s_{k+1}-s_{k}\right)^{R}+\left[g_{k}^{N}\right]^{T}\left(s_{k+1}-s_{k}\right)^{N} .
$$

Now observe that $x_{k} \in \mathcal{N}\left(X_{*}, 2 \delta\right)$ for $k$ large enough, the criticality of all points in $X_{*}$ and Lemma 12 guarantee the limit

$$
\lim _{k \rightarrow \infty} g_{k}^{N}=0
$$


and also, together with (16), (3), and (23), that the nonzero components of both $g_{k}^{R}$ and $\left(s_{k+1}-s_{k}\right)^{R}=\left(x_{k+1}+s_{k+1}-\left(x_{k}+s_{k}\right)\right)^{R}$ have the same sign. Hence we obtain that

$$
\left[g_{k}^{R}\right]^{T}\left(s_{k+1}-s_{k}\right)^{R} \geqq 0,
$$

and therefore that

$$
\begin{aligned}
g_{k}^{T}\left(s_{k+1}-s_{k}\right) & \geqq-\left|\left[g_{k}^{N}\right]^{T}\left(s_{k+1}-s_{k}\right)^{N}\right| \\
& \geqq-\sigma_{1} \beta_{2}\left\|g_{k}^{N}\right\|\left(\Delta_{k+1}+\Delta_{k}\right) \\
& \geqq-\frac{2 \sigma_{1} \beta_{2}}{\gamma_{0}}\left\|g_{k}^{N}\right\| \Delta_{k+1},
\end{aligned}
$$

where we have used (26), the Cauchy-Schwarz inequality, $\{13\}$, (AS.3), and $\{24\}$. Using the same equations, (17), $\{30\}$, and $\{33\}$, we also obtain that

$$
\left|s_{k+1}^{T} B_{k+1} s_{k+1}-s_{k}^{T} B_{k} s_{k}\right| \leqq \beta_{2}^{2}\left(b_{k+1} \Delta_{k+1}^{2}+b_{k} \Delta_{k}^{2}\right) \leqq \frac{2 \beta_{2}^{2}}{\gamma_{0}^{2}} b_{k+1} \Delta_{k+1}^{2} .
$$

Combining (24), (29), and (30), we have that

$$
m_{k+1}\left(x_{k+1}+s_{k+1}\right)-m_{k}\left(x_{k}+s_{k}\right) \geqq-\left[\frac{2 \sigma_{1} \beta_{2}}{\gamma_{0}}\left\|g_{k}^{N}\right\|+\frac{\beta_{2}^{2}}{\gamma_{0}^{2}} b_{k+1} \Delta_{k+1}\right] \Delta_{k+1} \text {. }
$$

The limits (27) and (14) then imply that, for a $k$ large enough and satisfying (16),

$$
m_{k+1}\left(x_{k+1}+s_{k+1}\right)-m_{k}\left(x_{k}+s_{k}\right) \geqq-\frac{1}{4} c_{3} \varepsilon^{2} \Delta_{k+1} .
$$

On the other hand, since $k+1 \in S$ and using (9), (14), and Lemma 6, we deduce that, for such a $k$,

$$
f\left(x_{k+1}\right)-m_{k+1}\left(x_{k+1}+s_{k+1}\right) \geqq \frac{1}{2} c_{3} \varepsilon^{2} \Delta_{k+1},
$$

and hence that

$$
\begin{aligned}
f\left(x_{k}\right)-m_{k}\left(x_{k}+s_{k}\right) & =f\left(x_{k+1}\right)-m_{k+1}\left(x_{k+1}+s_{k+1}\right)+m_{k+1}\left(x_{k+1}+s_{k+1}\right)-m_{k}\left(x_{k}+s_{k}\right) \\
& \geqq \frac{1}{4} c_{3} \varepsilon^{2} \Delta_{k+1} \\
& \geqq \frac{1}{4} c_{3} \gamma_{0} \varepsilon^{2} \Delta_{k} .
\end{aligned}
$$

But again the inequality $b_{k} \geqq 1,\{107\}$, and (34) yield that

$$
\left|\rho_{k}-1\right| \leqq \frac{2 \beta_{2}^{2}\left(c_{5} \sigma_{1}^{2}+1\right)}{c_{3} \gamma_{0} \varepsilon^{2}} b_{k} \Delta_{k},
$$

for $k$ sufficiently large and satisfying (16). The bound (18) then again implies that $\rho_{k}>\mu$ and $k \in S$ for $k$ large enough, which contradicts (16). The conditions (16) are thus impossible for $k$ sufficiently large, and all iterates are eventually successful. But this again contradicts (15). Hence our assumption (8) is false, and we obtain from (7) that there exists a subsequence $\left\{k_{t}\right\} \subseteq\left\{k_{j}\right\}$ such that $I\left(x_{k_{t}}+s_{k_{t}}\right)=I\left(X_{*}\right)$ for all $t$. Lemma 13 finally gives (1).

3. Acknowledgments. The authors thank J. J. Moré and G. Toraldo for pointing out the error considered here, and M. Lescrenier and A. Sartenaer for their comments on the present correction.

\section{REFERENCE}

[1] A. R. CONN, N. I. M. Gould, AND PH. L. ToInt, Global convergence of a class of trust region algorithms for optimization with simple bounds, SIAM J. Numer. Anal., 25 (1988), pp. 433-460. 